\title{
El poder de la autoeficacia en la mejora de la salud psicosocial de la persona teletrabajadora*
}

Eva Cifre \& Marisa Salanova

\author{
Universitat Jaume I Castellón, España
}

Recibido: 8 de mayo del 2012 / Aprobado: 1 de junio del 2012

Siguiendo el marco teórico de la psicología ocupacional positiva (POP) se ha adaptado la vía positiva del modelo de recursos-experiencias/emocionesdemandas (RED) (Salanova, Cifre, Martínez \& Llorens, 2007) en una muestra de teletrabajadores. Los resultados de regresiones moderadas evidencian la importancia de los recursos organizacionales (facilitadores, gestión de la distancia) y personales (personalidad, competencia mental) en relación con diferentes experiencias/emociones del teletrabajador (engagement, compromiso organizacional, interferencia y apoyo de la familia al trabajo). Las implicaciones teóricas y aplicadas se discuten al final del trabajo.

teletrabajo / autoeficacia / salud psicosocial

The power of self-efficacy in the improvement of psychosocial health in the teleworker

From the theoretical framework of Positive Occupational Psychology (POP) a $n$ adaptation of the positive model of Resources-Experiences/EmotionsDemands (RED, Salanova, Cifre, Martinez y Llorens, 2007) was utilize on a sample of teleworkers. The results of moderate regressions show the importance of organizational resources (facilitators, distance management) and personal resources (personality, mental competency) in relation to the different experiences/ emotions) of the teleworker (engagement, organizational commitment, family interference and support). Theoretical and applications implications are also discussed.

telework / self-efficacy / psychosocial health

\footnotetext{
* Esta investigación ha sido posible gracias a la ayuda del Ministerio Español de Economía y Competitividad (\#PSI2011-22400).

Correos electrónicos: cifre@uji.es; marisa.salanova@psi.uji.es
} 
Internet, telefonía móvil con MP4, ordenadores portátiles con Wi-Fi, tabletas..., son ejemplos de que las tecnologías de la información y la comunicación (TIC) nos rodean para, en principio, facilitarnos la calidad de vida en esta llamada sociedad de la información, o lo que Norbert Bilbeny (1993) ha denominado tercera revolución industrial.

Una nueva forma de plantear las relaciones laborales en esta nueva sociedad de la información es el teletrabajo. Al parecer, el origen del término data de principios de la década de 1970. De este modo, Nilles (1973) definió la actividad de "trabajar a distancia" como telecommuting, que en castellano puede traducirse por "teledesplazamiento", haciendo referencia a que es el trabajo el que se desplaza hasta el trabajador, y no viceversa. Desde entonces, el término telecommuting se ha empleado normalmente en Estados Unidos, mientras que en Europa se usa la palabra telework (teletrabajo) (Escobar, Jiménez, Del Riego \& Rodríguez, 1998).

De entre las diferentes definiciones de teletrabajo una de las clásicas es aquella que se le entiende como un modo de empleo en el cual el trabajador está localizado en un lugar remoto de una oficina central o de un centro de producción, en contacto con cotrabajadores o sin él, pero que permite la comunicación mediante el uso de la alta tecnología de los sistemas de comunicación (Conner, Fletcher, Firth-Cozens, \& Collins, 1994). 
Como refiere Padilla (1997), el teletrabajo es una estrategia funcional de una organización, basada en trabajar cuando quiera y en cualquier parte, haciendo por tanto los recursos humanos más flexibles.

De hecho, aunque cuesta reconocerse como personas teletrabajadoras (para simplificar utilizaremos el término "teletrabajadores" a partir de este momento), las prácticas de teletrabajo en sí -es decir, no estar el tiempo completo de nuestra jornada laboral en la oficina sino conectado mediante alguna TIC, alargando así incluso la jornada de trabajo "oficial"- son cada vez más habituales en nuestro contexto (Cifre, 2008). Algunas de las ventajas que potencialmente muestra el teletrabajo pasan por hacer más accesible el empleo a determinados colectivos como los minusválidos, las personas con residencia habitual en zonas rurales con dificultades de acceso a las grandes ciudades, los padres, y principalmente las madres de familias numerosas, como muestra el portal de la Asociación Española de Familias Numerosas; o a colectivos más generales, por las ventajas potenciales que conlleva el poder trabajar en cualquier momento y en cualquier lugar (por ejemplo, el de facilitar la posibilidad de conciliar la vida personal y la vida laboral).

\section{Teletrabajo y SALUd PSICOSOCIAL}

De los diversos temas relacionados con el teletrabajo, la salud psicosocial de los teletrabajadores es un aspecto que ha preocupado desde el principio a los investigadores, sobre todo del área de la ergonomía y la psicosociología aplicada. Además, desde un punto de vista más concreto, no es de extrañar que a raíz de esta nueva situación aparezcan nuevas necesidades instrumentales para la gestión y la organización, así como una regulación legal de las relaciones trabajo-empresa-sociedad en general, que en cuanto a la salud, en el contexto español, está recogida en el marco legislativo de la Ley de Prevención en Riesgos Laborales en España (Ley 31/1995, del 8 de noviembre).

En este sentido, las autoridades en materia de salud, tanto en el nivel estatal español como en el ámbito europeo, han dejado ya patente su preocupación al respecto en diversos documentos, por ejemplo en la Nota Técnica de Prevención 412 del Instituto Nacional de Seguridad e Higiene en el Trabajo en 1996, en España, sobre los criterios de implantación del teletrabajo (Pérez, Sancho, \& Nogareda, 1996); o los acuerdos aparecidos en el Boletín Informativo del 2001 de la Agencia Europea para la Seguridad y la Salud en el Trabajo a nivel europeo, entre los agentes sociales en los sectores de las telecomunicaciones y el comercio sobre las líneas directrices para el teletrabajo, incluidos los elementos relativos a la seguridad y la salud.

Es evidente la necesidad de innovar herramientas desde las ciencias huma- 
nas y de la salud para nuevas medidas preventivas y paliativas en busca de una salud integral del teletrabajador, como ocurre en otros contextos laborales donde ya están instauradas. Con estos cambios en la organización del trabajo, se van a producir nuevas necesidades y demandas, así como riesgos asociados. Y está claro que la psicología y la ergonomía tienen mucho que decir en apoyo a la gestión y el desarrollo de los recursos humanos en la empresa.

Sin embargo, los estudios empíricos sobre la implantación del teletrabajo son todavía escasos, y centrados principalmente en un punto de vista de la gestión organizacional, considerando de este modo los factores de gestión que pueden afectar la decisión de implantar o no el teletrabajo, tales como la percepción de los directores de recursos humanos (Pérez, Martínez \& De Luis, 2003; Pérez, Sánchez \& De Luis, 2002), así como las políticas, flexibilidad, preferencias y estrategias de comunicación de las empresas (Ilozor \& Ilozor, 2002; Martínez-Sánchez, Pérez-Pérez, De Luis-Carnicer \& VelaJiménez, 2007; Peters, Tijdens \& Wetzels, 2003).

En relación con la salud de los teletrebajadores, la mayor parte de los estudios se han centrado en el impacto del teletrabajo en la salud física y ergonómica (véase Alonso \& Cifre, 2002, para una ampliación del tema). Sin embargo, existe todavía escasez de estudios empíricos que analicen los factores psicosociales que puedan estar afectando la salud mental (bienestar psicosocial) de dichos teletrabajadores. Un trabajo destacable en este sentido en el contexto español es el realizado por Cifre, Salanova y Rodríguez (2006), donde comparando una muestra de teletrabajadores frente a trabajadores tradicionales, encontramos que la salud psicosocial de los primeros era superior a la de sus compañeros de oficina tradicional a partir de diferentes indicadores de salud psicosocial. Sin embargo, los teletrabajadores también mostraron un nivel de adicción al trabajo superior al de sus compañeros no teletrabajadores.

En este sentido, podríamos entender que el teletrabajo se puede convertir en un arma de doble filo para la conciliación de la vida personal y la vida laboral (Lewis, 2003), con potencial tanto positivo como negativo, ya que a veces gestionar al mismo tiempo y con la misma energía las demandas familiares y las laborales puede causar problemas a los empleados/as (Tausig \& Fenwick, 2001), sobre todo si generan adicción al trabajo, con las dificultades que ello conlleva para la conciliación. Sin embargo, en un estudio empírico realizado en Suecia (Hartig, Kylin \& Johansson, 2007) con empleados públicos que teletrabajaban al menos un $20 \%$ de su tiempo para, principalmente, evitar el traslado al trabajo y atender las demandas parentales, se vio que para los teletrabajadores el hogar seguía siendo un lugar de restablecimiento del estrés 
causado por las demandas laborales, aunque existían diferencias según el sexo (a las mujeres teletrabajadoras les costaba más restablecerse, y a los hombres teletrabajadores menos).

Teniendo en cuenta la investigación previa, el objetivo de este trabajo es, de acuerdo con el movimiento de la psicología positiva, estudiar los factores que pueden contribuir a la mejora y maximización de la salud mental de los teletrabajadores.

Para conseguir este objetivo, se realizó un estudio empírico con una muestra de teletrabajadores, basándose en la vía positiva del modelo RED (recursosemociones/experiencias-demandas) adaptado al teletrabajo (Salanova, Cifre, Martínez \& Llorens, 2007).

\section{RED: Un modelo comprehensivo de} la salud psicosocial en el trabajo y el teletrabajo

Partiendo de modelos anteriores sobre la salud psicosocial en el trabajo -en concreto, el modelo demandascontrol de Karasek (1979), el modelo demandas-control-apoyo social de Karasek y Theorell (1990), el modelo de demandas-recursos laborales de Demerouti, Bakker, Nachreiner y Schaufeli (2001), el modelo del proceso dual de Schaufeli y Bakker (2004), el modelo RED (Salanova et al., 2007) - se señala que es el ajuste/desajuste entre las demandas del puesto y los recursos personales/organizacionales los que llevarán a la percepción de un mayor bienestar (emociones positivas, engagement, compromiso organizacional, satisfacción) o malestar (burnout, tecnoestrés, adicción al trabajo) en el trabajo, lo cual a su vez influirá en consecuencias organizacionales (desempeño, absentismo/presentismo). Sin embargo, lo que caracteriza este modelo es la importancia concedida a las creencias de eficacia específica como recurso personal enmarcado en la teoría cognitiva social de Albert Bandura (2002).

La autoeficacia es, entre los recursos individuales, un elemento clave en este proceso de optimización de la salud psicosocial. Bandura (2002) la define como "[...] las creencias en las capacidades propias para organizar y ejecutar el curso de acciones requeridas para manejar las posibles situaciones" y tiene un carácter futuro. El modelo RED considera la autoeficacia como un antecedente de la percepción de ajuste/desajuste entre las demandas y los recursos: dependiendo de su nivel de autoeficacia, los trabajadores percibirán el contexto social de trabajo de manera diferente. Por lo tanto, consideramos, de acuerdo con el modelo RED, que la autoeficacia es un recurso personal fundamental por su poder modulador. Esto es así porque, según Schwarzer (1999), la autoeficacia puede marcar diferencias en la forma de pensar, sentir y actuar de las personas. De este modo, y en relación con los sentimientos/emociones, una autoeficacia baja está asociada con depresión, an- 
Figura 1

Vía positiva del modelo RED adaptado al teletrabajo

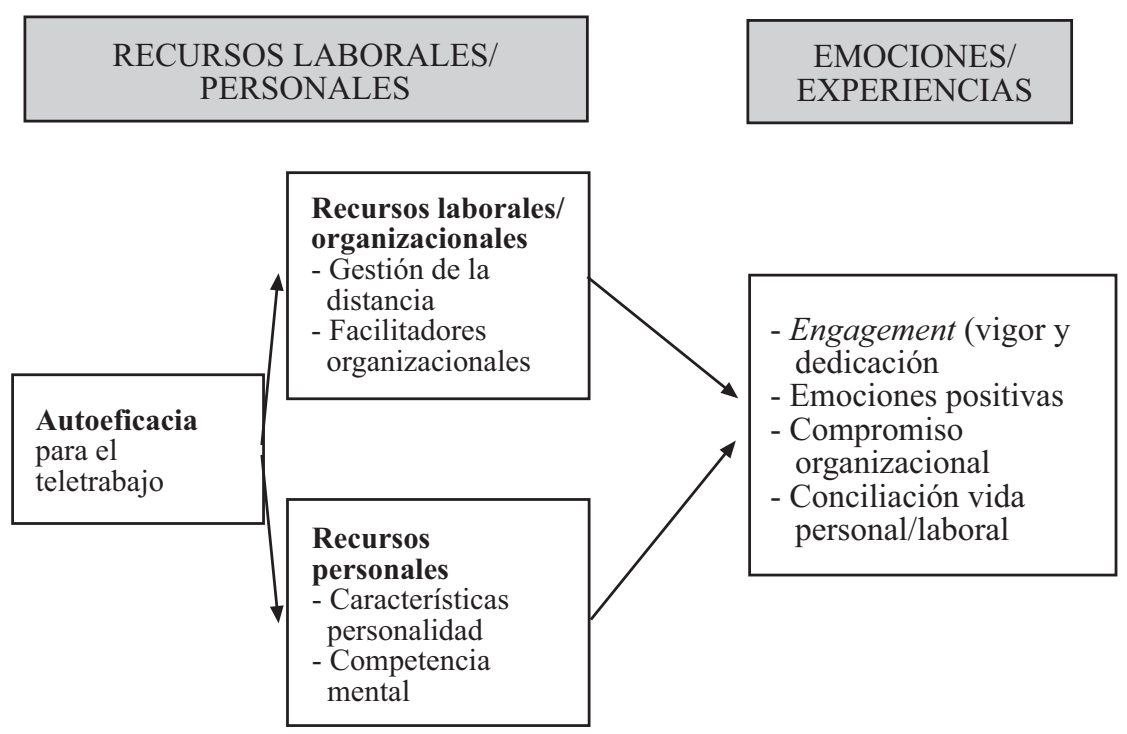

Nota: Variables incluidas en el cuestionario RED-TT.

siedad y desamparo (Grau, Salanova \& Peiró, 2000).

Además, y siguiendo la línea actual de la psicología ocupacional positiva (POP), que enfatiza la importancia de centrarnos en los aspectos más positivos del trabajo y la salud psicosocial, y no tanto en los negativos (por ejemplo, el estrés) como había ocurrido hasta ahora, el modelo que planteamos en el presente estudio recoge solo la vía positiva (recursos personales/organizacionales, bienestar psicológico, consecuencias positivas para la conciliación de la vida personal y laboral), o vía motivacional, del mencionado modelo RED. De este modo, la adaptación del modelo RED al teletrabajo presentado en este estudio quedaría tal y como se muestra en la figura 1.

Esta adaptación se ha hecho siguiendo dos criterios: 1) seleccionando la vía positiva del modelo RED (es decir, sin las demandas y sin las variables relacionadas con el malestar psicológico o estrés), y 2) seleccionando aquellas variables que la literatura ha mostrado como las más importantes en el contexto de teletrabajo, sobre todo las que hacen referencia a los recursos laborales/organizacionales y personales, que han sido los más estudiados por la literatura.

En relación con estas variables específicas del teletrabajo, cabe destacar como recursos laborales/organizacio- 
nales, en primer lugar, la importancia de la existencia de facilitadores, entendidos como aspectos organizacionales que contribuyen a la resolución de obstáculos (Martínez \& Salanova, 2005). Son las acciones y las estrategias dirigidas a mitigar los problemas ocasionados por los obstáculos que pueden interferir en la actuación (Tesluk \& Mathieu, 1999), y como ocurre con los obstáculos, pueden ser de naturaleza técnica o social (Schneider, White \& Paul, 1998). Además, según la literatura (véase, por ejemplo, Schneider \& Bowen, 1993) los facilitadores organizacionales mejoran el bienestar psicológico. Estos facilitadores deben ser, por tanto, específicos para el contexto donde se quieren analizar. En esta línea, y siguiendo la técnica de incidentes críticos de Flanagan (1954), Cifre, Salanova, Martínez-Pérez, Martínez, Llorens y Grau (2004b) encontraron una serie de facilitadores organizacionales para el teletrabajo que también agruparon en facilitadores técnicos y sociales para teletrabajar. Estos facilitadores se encuentran presentes en el teletrabajo para subsanar los obstáculos específicos con los que el teletrabajador se puede encontrar para llevar a cabo de manera habitual sus tareas, como por ejemplo los obstáculos tecnológicos, que han aparecido como obstáculos clave en experiencias piloto de teletrabajo (Salanova, Cifre, Martínez \& Llorens, 2007).

Por otro lado, hay autores como
Raghuram, Garud, Wiesenfeld y Gupta (2001), que indican la existencia de unos mecanismos que la organización puede poner en marcha para garantizar, en la medida de lo posible, el ajuste del teletrabajador a su nuevo puesto de trabajo. Estos mecanismos de gestión efectiva de la distancia (GED) pueden dividirse entre mecanismos de gestión estructurales (diseño de puestos y claridad de los criterios de evaluación del rendimiento) y mecanismos de gestión relacionales (creación de confianza empleados virtuales-compañeros y conexión organizacional, asegurándose de que estos teletrabajadores reciban toda la información relevante clave para sus carreras, al igual que el resto de sus compañeros "presenciales"). Dichos mecanismos recogerían los factores clave para el éxito en la implantación de programas de teletrabajo: comunicación, apoyo y confianza (Kowalski \& Swanson, 2005).

Entre los recursos personales del teletrabajador, cabe señalar que la literatura ha destacado el hecho de que poseer determinadas características de personalidad puede hacer a un candidato más o menos idóneo para el puesto de teletrabajador. Por ejemplo, Hernández-Muñoz (1997) destaca que el teletrabajador debe tener capacidad para organizar su propio trabajo, estar automotivado para la ejecución del trabajo y poseer autocontrol, orientarse a los resultados, tener capacidad de síntesis, sentir familiaridad y comodi- 
dad frente a su trabajo (no acaba de ser contratado o promocionado), mantener una buena relación profesional con su supervisor inmediato, disponer de un espacio adecuado para el trabajo en casa y evitar que la familia lo distraiga, prescindir, en cierto grado, de la vida social de la empresa, estar en capacidad de cambiar de horario y de lugar de trabajo (flexibilidad), y ser técnicamente autosuficiente. Además, cabe destacar la importancia de la competencia mental, al tratarse de puestos de trabajo cuyas tareas son principalmente cognitivas.

En relación con la autoeficacia, y de acuerdo con la teoría cognitiva social de Bandura (2002), debe ser específica para el contexto donde es evaluada, y por tanto, las escalas de medida se deberán adaptar al dominio particular de funcionamiento objeto de interés. Esto es así porque según el autor el sistema de creencias de eficacia no es un rasgo global, sino un grupo de creencias ligadas a ámbitos de funcionamiento diferenciados. Una persona puede considerarse muy eficaz ante ciertas situaciones y totalmente ineficaz en otras, dependiendo de los requerimientos situacionales (Martínez \& Salanova, 2006). En este caso consideramos fundamental la evaluación de la autoeficacia para el teletrabajo.

Finalmente, y en cuanto a las experiencias/emociones en el teletrabajo, consideramos dos tipos principales de variables que se deben analizar. Por un lado, consideramos que la mayor parte de las experiencias/emociones positivas señaladas por el modelo RED (engagement, emociones positivas, compromiso organizacional) tienen cabida en la adaptación de dicho modelo para el teletrabajador. Una de las experiencias potencialmente positivas que ofrece el teletrabajo es la posibilidad de conciliar la vida personal y la vida laboral. De hecho, el teletrabajo es ofrecido por las empresas como una de las estrategias flexibles que permiten dicha conciliación (véase Cifre \& Salanova, 2004). Así, para el teletrabajador, las ventajas del teletrabajo suelen asociarse con un incremento en la flexibilidad horaria, que permite la ejecución de este según un plan independiente e individualizado al tiempo y necesidades de cada uno (Pérez et al., 1996). Esta flexibilidad, además de algunos de los riesgos mencionados anteriormente (adicción al trabajo, cambio en los biorritmos) es vista principalmente como una ventaja por parte del teletrabajador, ya que le permite potencialmente una mayor posibilidad de conciliar su vida personal (apreciada principalmente en mujeres con hijos pequeños) con su vida laboral.

Pero como se ha señalado anteriormente, estas ventajas que ofrece el teletrabajo de cara a la conciliación de la vida personal con la familiar se ha visto que puede ser una espada de doble filo, con potencial tanto positivo como negativo (Lewis, 2003), ya que a veces al tener que gestionar al mismo 
tiempo y con la misma energía las demandas familiares y laborales se corre el riesgo de causar problemas a los empleados/as (Tausig \& Fenwick, 2001). Por ello, consideramos fundamental evaluar en este caso tanto la conciliación como la interferencia de la vida personal y la vida laboral.

Por ello, se ha llevado a cabo un estudio empírico cuyo objetivo es poner a prueba una adaptación del modelo RED al teletrabajo, teniendo en cuenta únicamente la vía positiva de dicho modelo. Al respecto, las hipótesis planteadas son las siguientes:

- Hipótesis 1.- Los recursos laborales/ organizacionales y personales estarán asociados positivamente con las experiencias/emociones positivas en el teletrabajo.

- Hipótesis 2.- Los altos niveles de autoeficacia para teletrabajar se asociarán significativamente con los altos niveles de experiencias/emociones positivas en el teletrabajo.

- Hipótesis 3.- La autoeficacia para teletrabajar modulará la relación entre los estresores laborales/individuales y sus consecuencias. Es decir, se espera que los facilitadores organizacionales, las estrategias de gestión efectiva de la distancia (GED), las características de personalidad y la competencia mental se asocien positivamente con las experiencias/ emociones positivas en el teletrabajo solo en aquellos teletrabajadores con niveles elevados de autoeficacia para el teletrabajo (AET).

\section{Metodología}

\section{Procedimiento y participantes}

Siguiendo con la filosofía de esta nueva forma de empleo, el modo de recogida de muestra ha sido on-line. Para ello, se solicitó permiso para la utilización de un instrumento específico para la evaluación de los riesgos psicosociales del teletrabajo: el RED-TT (Cifre et al., 2004b) y se insertó en la página web del equipo de investigación WoNT (http://www.wont.uji.es), al cual pertenecía el cuestionario. Posteriormente, se publicitó la cumplimentación del cuestionario, enfatizando sus ventajas - principalmente el feedback inmediato y on-line que se obtiene sobre los riesgos psicosociales de su teletrabajo- a través de asociaciones de teletrabajadores, de asociaciones y colegios profesionales que cuentan con un elevado número de teletrabajadores (traductores, periodistas, etcétera), así como a través de portales que la agencia de publicidad contratada supuso que le daría mayor difusión. Una vez limpiada la base de datos, las variables objeto de estudio fueron proporcionadas por los autores del cuestionario para la realización de este trabajo. Un total de 100 teletrabajadores, con un rango de edad entre 22 y 62 años, y una media de 38 años (DT=11.41) participaron en el estudio. Llevaban una media de 33.2 
meses teletrabajando (DT=35.52). De ellos, $54 \%$ eran hombres y $65 \%$ tenía al menos un hijo.

\section{Medidas}

Todas las escalas utilizadas en el presente estudio (facilitadores para el teletrabajo, GED, características de personalidad del teletrabajador, competencia mental, autoeficacia para el teletrabajo, engagement, emociones, compromiso organizacional, interferencia y apoyo familia-trabajo y trabajo-familia) son escalas autoconstruidas, pertenecientes al cuestionario on-line RED-TT (Cifre et al., 2004). La escala de respuesta oscila en todos los casos entre 0 (Totalmente en desacuerdo/Nunca) a 6 (Totalmente de acuerdo/Siempre).

a) Variables independientes: Recursos laborales/organizacionales e individuales

La escala de facilitadores para el teletrabajo está compuesta por ocho ítems, que recogen tanto los facilitadores técnicos como los sociales. A los teletrabajadores se les pedía que indicaran en qué medida habían sido importantes los facilitadores que se listaban a continuación en superar los obstáculos en su teletrabajo. Un ejemplo de facilitador es: "Utilización de diferentes estrategias para no ser interrumpido en las horas de trabajo por llamadas personales, tales como activar diferentes líneas telefónicas, utilizar el contestador, tener un teléfono móvil solo de trabajo,...". El coeficiente alfa de Cronbach es de .87.

La escala de gestión efectiva de la distancia (GED) estaba compuesta originalmente por nueve ítems que constituían cuatro subescalas: independencia (2 ítems), claridad de criterio (2 ítems), conexión organizacional (2 ítems), y confianza interpersonal (3 ítems). Tras la realización de diversos análisis estadísticos (que incluían análisis factoriales con todos los ítems, análisis de mejora de fiabilidad de la escala....), y aunque la escala presenta un alfa de Cronbach bastante ajustado (.61), se decidió conservar todos los ítems para preservar la filosofía de su construcción original (siguiendo los mecanismos de GED, de Raghuram et al., 2001). Un ejemplo de ítem para esta escala es: "Desde que teletrabajo, tengo claros los criterios de evaluación de mi trabajo por parte de mi organización".

La escala de caracteristicas de personalidad idóneas para el teletrabajo estaba compuesta por siete ítems que recogían las capacidades de: resolución de problemas, toma de decisiones, adaptabilidad, autoplanificación, autodisciplina, autoevaluación y automotivación. Un ejemplo de ítem es: "En mi teletrabajo soy capaz de planificar por mí mismo las tareas a corto y medio plazo". El alfa de Cronbach de la escala es de .91 .

La escala de competencia mental estaba compuesta por tres ítems. Un ejemplo de ítem es: "En mi teletrabajo 
soy capaz de estar pendiente y recordar muchas cosas a la vez". El alfa de Cronbach de la escala es de .92 .

b) Variable moduladora: La autoeficacia para el teletrabajo (AET)

La escala de AET estaba compuesta por cinco ítems. Un ejemplo de ítem es: "Soy capaz de teletrabajar aunque tenga que resolver problemas técnicoprofesionales difíciles derivados de su uso". El alfa de Cronbach de la escala es de .97 .

\section{c) Variables dependientes: Emociones/ experiencias positivas en el teletrabajo}

La variable de engagement fue evaluada a través de las dos escalas consideradas como el "corazón del engagement": las escalas de vigor y de dedicación. La escala de vigor en el teletrabajo estaba compuesta por seis ítems. Un ejemplo de ítem es: "Cuando teletrabajo me siento lleno de energía". El alfa de Cronbach de la escala es de .93. La escala de dedicación en el teletrabajo estaba compuesta por seis ítems. Un ejemplo de ítem es: "Cuando teletrabajo aprendo cosas nuevas e interesantes". El alfa de Cronbach de la escala es de .96 .

La escala de emociones en el teletrabajo consistía en un diferencial semántico de Osgood compuesto por tres ítems. A los teletrabajadores se les preguntaba cómo se sentían generalmente cuando teletrabajaban, y debían elegir, en una escala de 0 a 6 , qué polo se acercaba más a su situación. Los polos entre los que debían elegir eran "Tenso/Agobiado-Relajado/Tranquilo", "Desanimado-Entusiasmado" y "A disgusto-A gusto". El alfa de Cronbach de la escala es de .81 .

La escala de compromiso organizacional estaba compuesta por tres ítems. Un ejemplo de ítem es "Los problemas de mi organización son 'mis' problemas". El alfa de Cronbach de la escala es de .84 .

La escala de interferencia del teletrabajo en la familia estaba compuesta por cuatro ítems. Un ejemplo de ítem es: "Estoy tan preocupado por asuntos de trabajo que me olvido de mis asuntos familiares". El alfa de Cronbach de la escala es también de .84 .

La escala de interferencia de la familia en el teletrabajo estaba compuesta por cuatro ítems. Un ejemplo de ítem es "Debido a mis obligaciones familiares estoy demasiado cansado como para realizar mi trabajo". El alfa de Cronbach de la escala es de .68.

La escala de apoyo del teletrabajo a la familia estaba compuesta por tres ítems. Un ejemplo de ítem es: "Cuando tengo problemas personales, en el trabajo puedo contar con mis compañeros". El alfa de Cronbach de la escala es de .80 .

La escala de apoyo de la familia en el teletrabajo estaba compuesta por seis ítems. Un ejemplo de ítem es: "Me 
siento escuchado y comprendido cuando hablo de trabajo con mi familia". El alfa de Cronbach de la escala es también de 71 .

\section{Análisis de datos}

En primer lugar, analizamos las consistencias internas (alfa de Cronbach), análisis descriptivos y correlaciones entre variables con el programa SPSS 17.0. En segundo lugar, llevamos a cabo análisis de regresión modulada, al ser el método recomendado para poner a prueba efectos de interacción (Cohen \& Cohen, 1983; Landsbergis, Schnall, Warren, Pickering \& Schwartz, 1994). Así, se realizaron diversos análisis de regresión múltiple jerárquica para detectar efectos principales y efectos de interacción de GED, los facilitadores, las características de personalidad, la competencia mental y la variable moduladora (autoeficacia para el teletrabajo) sobre cada una de las medidas de emociones/experiencias positivas en el trabajo estudiadas. Para poner a prueba los efectos de la interacción, se crearon términos multiplicativos de las variables independientes (Cohen \& Cohen, 1983; Kleinbaum, Kupper \& Muller, 1988). En este caso no se utilizaron coeficientes estandarizados al contar todas las escalas de medida con el mismo rango (de 0 a 6 ).

Las variables independientes entraron en la ecuación en cuatro pasos sucesivos (Aiken \& West, 1991; Jaccard, Turrisi \& Wan, 1990). En el primer paso
(1) se introdujeron las variables control: la edad, el sexo y el tiempo que llevaban teletrabajando; en el segundo paso (2) se introdujeron las variables independientes; en el tercer paso (3), la variable moduladora (AET); finalmente, en el cuarto paso (4) se introdujeron las interacciones de dos vías de las variables independientes multiplicadas por la variable moduladora (GED x AET, facilitadores x AET, características personalidad x AET, competencia mental $x$ AET). Los efectos de interacción significativos apoyarían la hipótesis 3 . No obstante, se tuvieron en cuenta también los efectos principales, ya que como señalan Jaccard et al. (1990) los efectos principales de las variables independientes generalmente son una pieza significativa de información. Los efectos principales significativos apoyarían la hipótesis 1. Por último, el efecto principal significativo de la AET apoyaría la hipótesis 2.

En total se realizaron ocho análisis de regresión múltiple jerárquica (uno por variable dependiente analizada). Para interpretar las variables estandarizadas a priori, en la tabla 2 se presentan los coeficientes de regresión no estandarizados (B) (Aiken \& West, 1991). Por último, los efectos de interacción significativos se representan gráficamente para una mejor interpretación de los resultados. Se siguió el procedimiento de Cohen \& Cohen (1983) y Jaccard et al. (1990). Específicamente, generamos, a partir de la ecuación de 
regresión, líneas de regresión separadas para representar la relación entre recur- sos y emociones/experiencias positivas en el teletrabajo a niveles relativamente

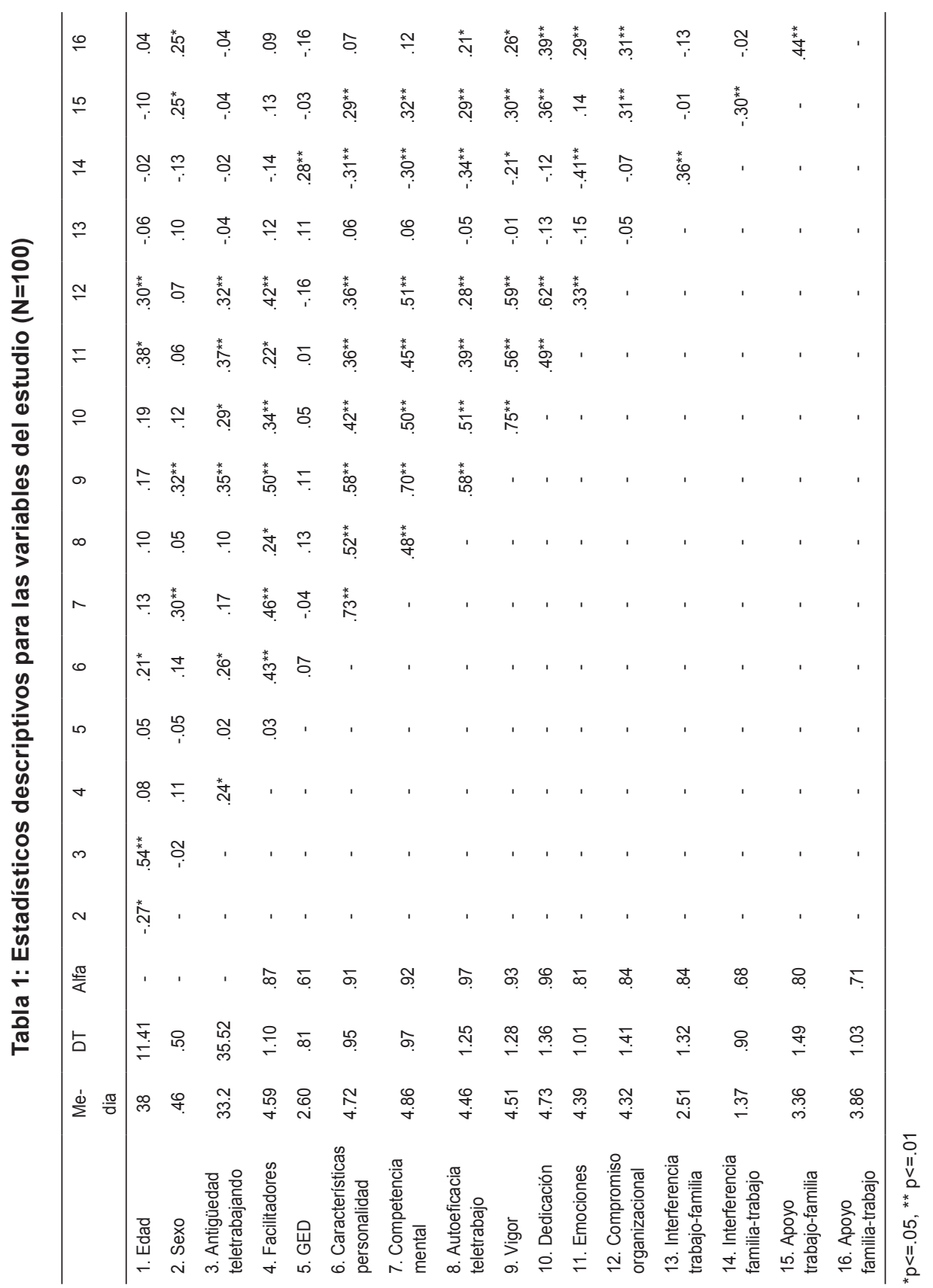


altos (Media $+1 \mathrm{DT})$ y relativamente bajos (Media - 1DT) de la variable moduladora (AET). Para ello se fijaron los valores tanto de la variable moduladora (AET) como de la variable independiente en cada una de las rectas. Posterior-

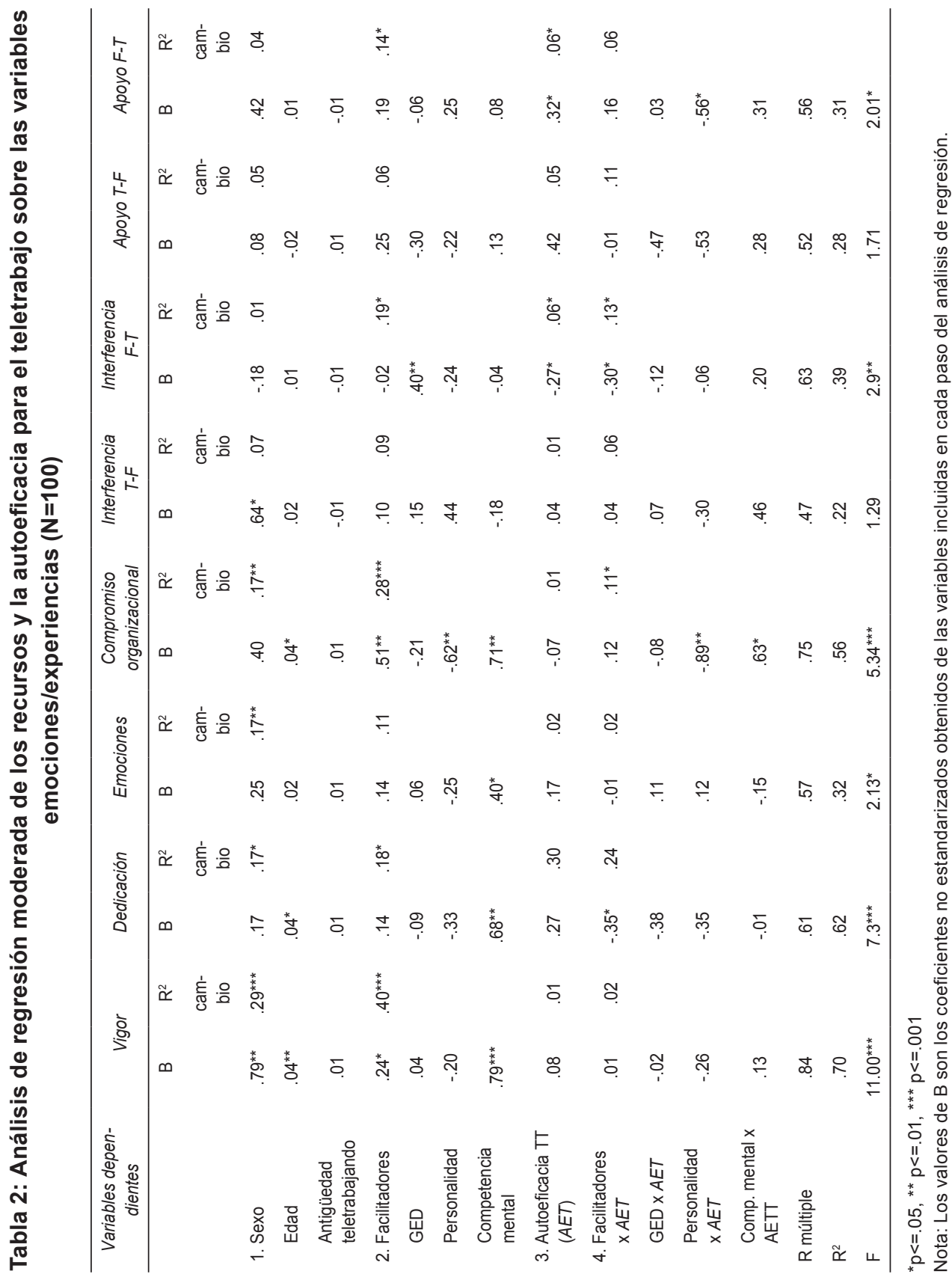


mente, se generaron líneas de regresión simples para introducir estos valores en la ecuación de regresión múltiple jerárquica, teniendo en cuenta el efecto de la interacción. La fórmula empleada para analizar e interpretar una interacción a dos vías fue:

$$
\mathrm{Y}=b_{0}+b_{1}(\mathrm{X})+b_{2}(\mathrm{Z})+b_{3}(\mathrm{XZ})+\mathrm{e}
$$

donde $b_{0}$ es la constante, $b_{1}$ es el coeficiente no estandarizado de la variable independiente, $b_{2}$ es el coeficiente no estandarizado de la variable moduladora, $b_{3}$ es el coeficiente no estandarizado resultante del producto de las dos variables recién mencionadas (término ya referido anteriormente), y el último término (XZ) es el producto de los dos primeros (véase Preacher, 2007, para una explicación más detallada del procedimiento).

\section{Resultados}

\section{Análisis descriptivos}

La tabla 1 muestra los descriptivos (medias, desviaciones típicas, alfas de Cronbach y correlaciones de Pearson de orden cero) de las variables consideradas en el estudio. Todos los coeficientes alfa muestran una consistencia interna razonable, superando en la mayoría de casos con holgura el .70 recomendado por Nunnaly y Bernstein (1994). Esto es así en todos los casos, excepto en el de la GED, en el cual supera ligeramente el valor de .60 .

De la matriz de correlaciones, cabe destacar, en primer lugar, que el sentido de las correlaciones coincide con el esperado en función de la literatura revisada (por ejemplo, la interferencia familia-trabajo correlaciona negativamente con seis variables, destacando su correlación positiva con la interferencia trabajo-familia; también es negativa la correlación entre la interferencia familia-trabajo y el apoyo trabajo-familia). Gran parte de las variables psicosociales están correlacionadas, excepto la variable interferencia trabajo-familia, que, como hemos mencionado, solo correlaciona positivamente con la interferencia familia-trabajo, y la variable GED, que solo correlaciona positivamente con la interferencia familia-trabajo.

\section{Test de hipótesis}

En la tabla 2 se muestran los resultados correspondientes a los análisis de regresión moderada realizada.

Los valores de la $\mathrm{F}$ de los modelos propuestos nos indican que ni la interferencia ni el apoyo del trabajo hacia la familia ni el apoyo de la familia hacia el trabajo son explicadas por las variables evaluadas en este estudio (recursos personales/organizacionales, y autoeficacia para el teletrabajo). Por otro lado, cabe señalar que tanto el vigor como la dedicación (dimensiones clave del engagement en el trabajo) presentan porcentajes de explicación muy elevados por los modelos propuestos $\left(\mathrm{R}^{2}=.70 \mathrm{y}\right.$ $\mathrm{R}^{2}=.62$, respectivamente). 
En el resto de emociones/experiencias se confirma la hipótesis 1 (H1) en la mayor parte de los casos (vigor, dedicación, compromiso organizacional e interferencia familia-trabajo), dado que son explicadas por al menos una de las variables predictoras propuestas de manera significativa, y suponiendo un cambio en la $\mathrm{R}^{2}$ entre el paso $1 \mathrm{y}$ el paso 2 a su vez significativo. De este modo, en el caso del vigor este se relaciona con una variable organizacional (facilitadores) y una personal (competencia mental); la dedicación solo con una personal (competencia mental); el compromiso organizacional con una organizacional (facilitadores); y las dos personales (personalidad, de manera inversa, y la competencia mental). Cabe destacar también la relación entre una variable organizacional
(GED) y la interferencia de la familia hacia el trabajo.

Precisamente, esta interferencia y apoyo de la familia hacia el trabajo son los únicos casos en los cuales se confirma la hipótesis 2 (H2), donde la autoeficacia para teletrabajar muestra ser un recurso personal clave, al presentar resultados negativos en un caso (a mayor autoeficacia, menor interferencia de la familia hacia el trabajo) y positivos en el otro (a mayor autoeficacia, mayor apoyo de la familia hacia el trabajo).

Finalmente, en relación con la hipótesis 3 (H3), que postulaba el efecto modulador de la autoeficacia para el teletrabajo sobre la relación del resto de variables con las emociones/experiencias resultantes del teletrabajo, los resultados del paso 4 nos indican que esta

Figura 2

Efecto de la interacción de dos vías de facilitadores organizacionales y autoeficacia para el teletrabajo sobre dedicación mientras teletrabaja

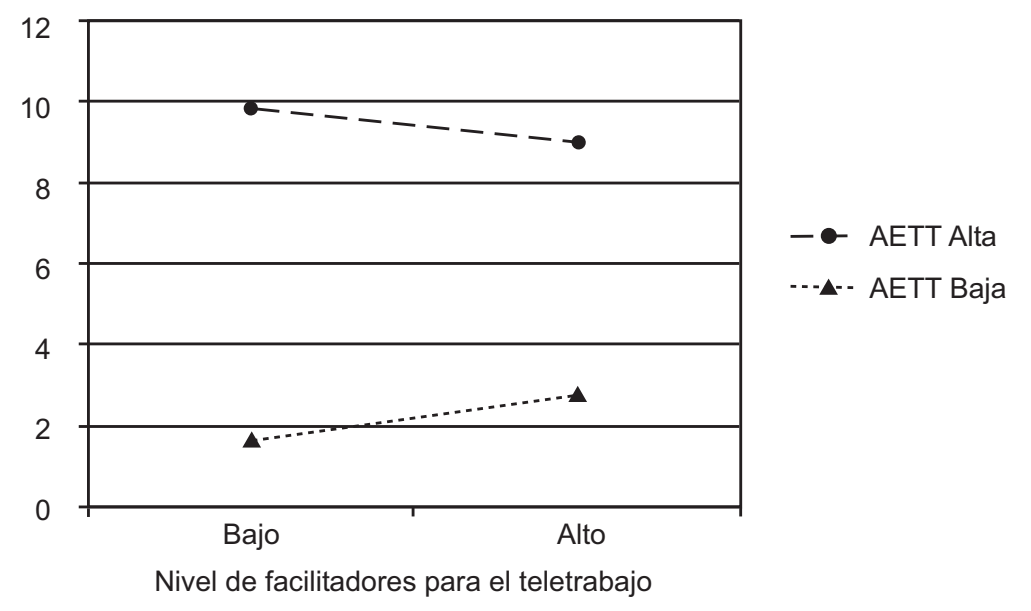


Figura 3

Efecto de la interacción de dos vías de características de personalidad idóneas para el teletrabajo y autoeficacia para el teletrabajo sobre el compromiso organizacional

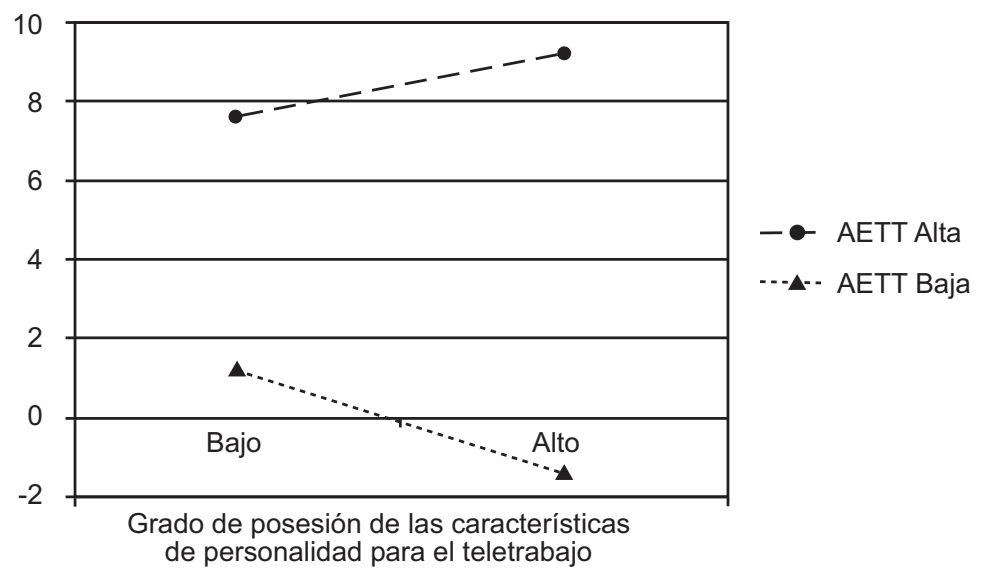

Figura 4

Efecto de la interacción de dos vías de competencia mental y autoeficacia para el teletrabajo sobre el compromiso organizacional

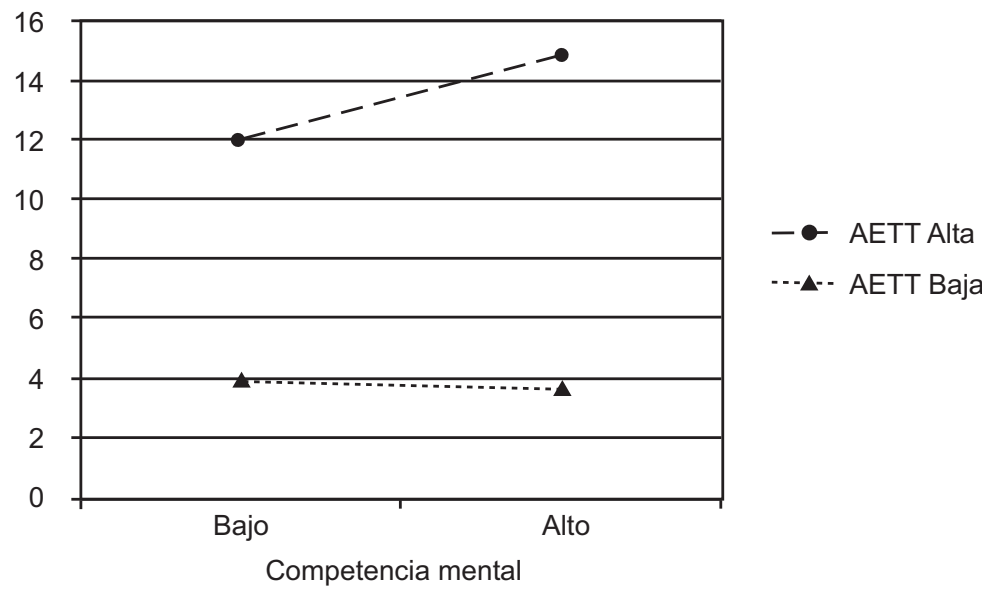

hipótesis se confirma en tres variables resultados concretas: dedicación, compromiso organizacional e interferencia de la familia hacia el trabajo.

En el caso de la dedicación, en la figura 2 se representa gráficamente el efecto de la interacción de los facilitadores con la AET, mostrando que los niveles de dedicación distan en un elevado grado en función al nivel de AET. Así, los teletrabajadores con una elevada AET poseen niveles de dedicación al teletrabajo mucho más eleva- 
dos que sus compañeros con una AET más baja. Además, el poseer más o menos facilitadores para el teletrabajo también los afecta de manera diferente: mientras que los teletrabajadores con baja AET necesitan facilitadores para sentirse más dedicados al trabajo, al grupo de alta AET le ocurre lo contrario.

Para el caso del compromiso organizacional, se indagó primero la interacción entre la AET con la personalidad idónea para el teletrabajo, y luego la interacción entre la AET y la competencia mental. Según el primer análisis (figura 3), las características de la personalidad no cumplen en el sentido de lo esperado en los teletrabajadores con alta AET, puesto que la interacción entre la AET y dichas características de personalidad se produce en sentido negativo con el com- promiso organizacional: las personas con alto grado de AET muestran niveles muy inferiores en compromiso organizacional respecto de sus compañeros con baja AET, disminuyendo además dicho compromiso a medida que sus características de personalidad se ajustan más a las hipotéticamente idóneas para el teletrabajo. Sin embargo, esto no ocurre en los teletrabajadores con baja AET: estos se comprometen más con el teletrabajo si sus características de personalidad (capacidad de automotivación, de autoplanificación) se ajustan a las idóneas de los teletrabajadores.

Por otro lado, y en cuanto a la competencia mental, las variables sí se relacionan en el sentido esperado (véase la figura 4). De este modo, los teletrabajadores que se perciben con poca AET muestran niveles bajos de compromiso

Figura 5

Efecto de la interacción de dos vías de facilitadores organizacionales y autoeficacia para el teletrabajo sobre la interferencia de la familia sobre el teletrabajo

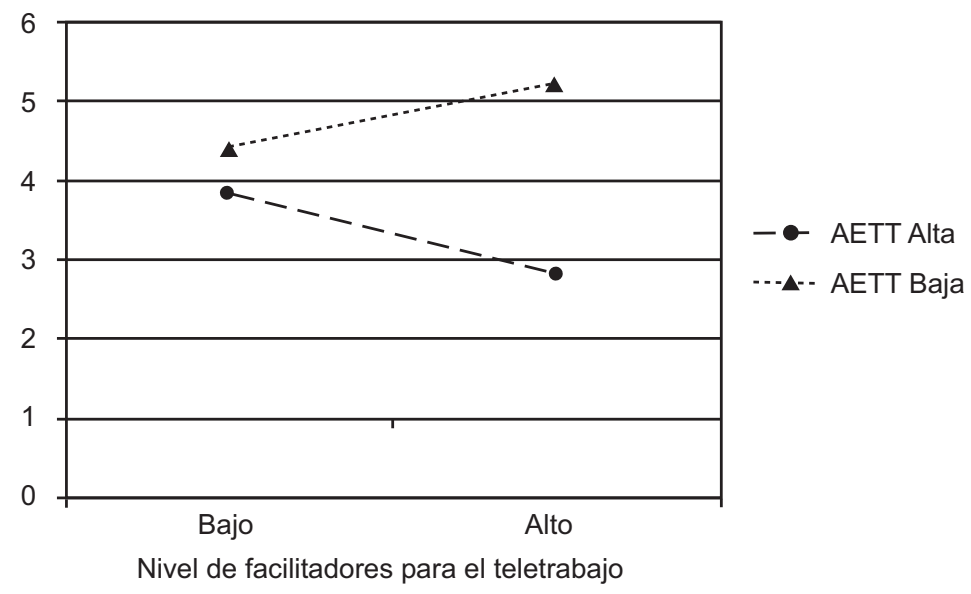


organizacional independientemente de su grado de competencia mental (básica para este tipo de trabajo), mientras que los teletrabajadores con una elevada $A E T$, además de mostrar niveles más elevados de compromiso organizacional, este se incrementará significativamente a medida que aumenta su competencia mental.

Finalmente, en el caso de la interferencia de la familia hacia el teletrabajo, los facilitadores organizacionales son los que interaccionan con la AET para predecir esta interferencia. Tal y como se muestra en la figura 5, en este caso, aunque el grado de interferencia de la familia hacia el trabajo es muy similar tanto para los teletrabajadores con alta AET como para los de baja AET cuando el nivel de facilitadores organizacionales es bajo, este comportamiento cambia cuando se incrementa el nivel de facilitadores para el teletrabajo. En este caso, los teletrabajadores con menor AET aumentan el grado de interferencia, mientras que los teletrabajadores con mayor AET consiguen disminuir dicha interferencia.

En lo que se refire al apoyo del teletrabajo a la familia, al igual que ocurría en el caso de la interferencia teletrabajo-familia, no parecen ser significativas ni las regresiones realizadas (en ninguno de sus pasos) ni los cambios en $\mathrm{R}^{2}$. Solo la AET aparece como significativa en el último paso de la regresión. Por tanto, podemos decir que solo la hipótesis 2 se confirma, aunque al aparecer tan solo como significativa en el último paso, podría tratarse de un resultado estadístico artifactual (aparece solo por la conjunción del gran número de variables incorporado en este caso).

En el caso del apoyo de la familia al teletrabajo también aparece una interacción significativa de las características de personalidad idóneas para el teletrabajo con la AET. Sin embargo, dado que la introducción de estas variables no suponen un incremento en la $\mathrm{R}^{2}$ del modelo explicado, consideramos que no debe tenerse en cuenta dicho resultado.

\section{Discusión}

El objetivo de este estudio ha sido poner a prueba empíricamente una adaptación de un modelo teórico comprehensivo de salud psicosocial en el trabajo, el modelo RED (recursos-emociones/experiencias-demandas) al teletrabajo, teniendo en cuenta únicamente la vía positiva de dicho modelo. Con ello se pretendía aplicar un modelo teórico a una realidad cada vez más cercana, como es la existencia de nuevas formas de trabajo facilitadas por algo ya tan cotidiano para la mayoría de nosotros como son las tecnologías de la información y la comunicación (TIC).

Para desarrollar este objetivo se han puesto a prueba tres hipótesis, dos de ellas vinculadas de forma muy cercana con la teoría cognitiva social de Bandura, al hipotetizar que la autoeficacia 
específica para el teletrabajo (AET) iba a jugar un papel clave a la hora de experienciar/sentir emociones en el teletrabajo. De este modo, además de hipotetizar que los recursos personales y organizacionales iban a tener una influencia directa sobre dichas experiencias/emociones estudiadas (tal y como predice el modelo RED y sus antecesores, como el modelo demandas/control, el modelo demandas/recursos, el modelo dual,...) (hipótesis 1) también se hipotetizó que la AET iba a tener influencia sobre dichas emociones/experiencias de dos maneras diferentes: de manera directa (hipótesis 2) o a través de su efecto modulador sobre los recursos que actuaban de variables independientes (hipótesis 3).

De este modo, en relación con la hipótesis 1 , los resultados indican que existe una serie de recursos tanto organizacionales/laborales como personales/individuales que van a afectar de manera directa a la forma en que los teletrabajadores se sientan mientras teletrabajan. Estos recursos son principalmente los facilitadores que la organización les proporcione para teletrabajar (afectan al engagement del teletrabajador, es decir, a los niveles de vigor y de dedicación en el teletrabajo; y al compromiso de los teletrabajadores con la organización para la que teletrabajan) y la competencia mental, recurso fundamental para este tipo de trabajo donde las tareas son mayoritariamente de índole cognitiva (afecta de manera directa el vigor, las emociones positivas y el compromiso organizacional).

Hemos comprobado la importancia de estos recursos, uno de carácter organizacional y otro individual, a la hora de predecir la manera como se pueden sentir los teletrabajadores respecto al teletrabajo. Los otros dos recursos analizados (también uno de cada índole), aunque han influido en algunas de las variables dependientes analizadas, su trascendencia ha sido mucho menor (en el caso de las características de personalidad idóneas para el teletrabajo o la gestión efectiva de la distancia). Por lo tanto, y en relación con la hipótesis 1 , podemos afirmar que este estudio pone de manifiesto la importancia de que los teletrabajadores cuenten con recursos organizacionales o personales adecuados para llevar a cabo su teletrabajo diario, para lo cual es fundamental dotarles (en caso de los recursos organizacionales) o seleccionarles (en caso de los recursos personales) en función a que los posean o no, para intentar garantizar así un mejor ajuste a esta nueva forma de trabajo.

Respecto a la hipótesis 2, la autoeficacia para el teletrabajo ha mostrado su influencia directa solo sobre la interferencia y el apoyo de la familia hacia el teletrabajo. Tal y como pronostica la teoría cognitiva social de Bandura (2002), la AET permite afrontar los obstáculos (interferencia de la familia en el teletrabajo) e incrementar los recursos (apoyo de la familia hacia el tele- 
trabajo) al influir en la forma de percibir la realidad del teletrabajador.

En relación con la hipótesis 3, esta hacía referencia al papel modulador de la AET entre una variable predictora (recurso) y las variables dependientes (emociones/experiencias) predichas. Este es el caso de las variables dedicación, compromiso organizacional y la interferencia y el apoyo de la familia en el teletrabajo.

En el caso de la dedicación, el hecho de que poseer o no facilitadores afecte de manera diferente a los teletrabajadores en función a que se perciban más o menos autoeficaces para teletrabajar (la dedicación disminuye en el primero de los casos y aumenta en el segundo) puede deberse a que los teletrabajadores más autoeficaces (con niveles mucho más elevados de dedicación) perciban los facilitadores como un recurso extra que no necesitan. Sin embargo, los teletrabajadores con menores niveles de AET sí aprecian estos facilitadores (un motivador externo a ellos) para poder llevar a cabo su teletrabajo, por lo que su nivel de dedicación se incrementa en este caso.

En el caso del compromiso organizacional, la AET modula entre los dos recursos individuales analizados (características de personalidad y competencia mental) y la variable predicha. En el caso de las características de personalidad idóneas para el teletrabajo, los resultados indican que, en contra de lo esperado, las personas con alto grado de AET muestran niveles muy inferiores en compromiso organizacional que sus compañeros con baja AET, compromiso que incluso va disminuyendo a medida que aumenta el ajuste de sus características de personalidad al teletrabajo, lo que no ocurre de nuevo en los teletrabajadores con baja AET. En el caso de los teletrabajadores con baja AET, podemos interpretar que el hecho de incrementar el ajuste de sus características de personalidad al teletrabajo hace que se incremente también su nivel de compromiso con la organización para la que teletrabajan, puesto que tienen la posibilidad de suplir las carencias de unas características con las otras. Esto es así porque, aunque le llamemos características de personalidad, muchas de ellas se pueden incrementar a través de cursos de formación ofrecidos por la misma organización (como la autoplanificación y la automotivación), que haga que estos teletrabajadores se sientan cada vez más comprometidos con el teletrabajo. Sin embargo, es posible que los teletrabajadores con alta AET y las características idóneas dejen de ver el teletrabajo como un reto (algo habitual en los trabajadores con alta autoeficacia: la búsqueda de situaciones retadoras en el trabajo), por lo que su nivel de compromiso disminuya. No obstante, este resultado requeriría mayor investigación utilizando muestras más amplias donde exista mayor variabilidad en características de personalidad, y además analizar cada una de ellas por 
separado en cuanto a su relación con el compromiso organizacional.

En cuanto al efecto modulador de la AET entre la competencia mental y el compromiso organizacional, los resultados revelan que mientras los teletrabajadores con elevada AET muestran elevados niveles de este compromiso, los que se incrementan incluso más a medida que aumenta su competencia mental, los teletrabajadores con baja AET muestran niveles casi estables (y muy bajos) en este compromiso. Esto se debería a que la competencia mental (como hemos comentado anteriormente) es una competencia básica para el teletrabajo, que es una actividad principalmente cognitiva. Así, quienes no la posean y no se sientan capaces de teletrabajar no se comprometerán con el teletrabajo. Sin embargo, quienes sí se ven capaces de teletrabajar (AET elevada) incrementarán aún más su compromiso si además cuentan con niveles elevados de esta competencia.

En relación con la interferencia de la familia con el teletrabajo, la AET modula su relación con respecto a la mayor o menor presencia de facilitadores organizacionales. Y modula en el sentido esperado: los teletrabajadores con mayor AET obtienen un mayor rendimiento de los facilitadores organizacionales que les ofrece su organización, consiguiendo así disminuir el grado de interferencia de la vida familiar/personal en la actividad del teletrabajo.

Finalmente, la AET ha mostrado modular la relación entre el grado en el que los teletrabajadores poseen las características de personalidad idóneas para teletrabajar y el apoyo que tienen de su familia para ello. En este caso, y en contra de lo que en un principio cabría esperar, son los teletrabajadores con menor AET los que perciben un mayor apoyo de la familia para realizar este tipo de labor, apoyo que se incrementa a medida que sus características de personalidad se ajustan más al teletrabajo. El caso contrario ocurre con los teletrabajadores con mayor AET: el apoyo de su familia, además de ser menor, disminuye a medida que aumenta el ajuste de sus características de personalidad al teletrabajo. Aunque en un principio parezca un resultado contradictorio, podemos pensar que la familia/entorno personal de los teletrabajadores con menor AET pero que deben teletrabajar, sienta que debe apoyarlo para que lleve a cabo sus tareas relacionadas con el teletrabajo. Por tanto, le relevan de las tareas domésticas para que pueda centrarse en el teletrabajo, les escuchan cuando necesitan hablar de este. Además, este apoyo se incrementa en la medida en que el entorno percibe que sí posee las características de personalidad idóneas del teletrabajador, para motivarle a continuar en la actividad. Sin embargo, en el caso de los teletrabajadores con un elevado nivel de AET, y que además tienen las características de una personalidad idónea, se concluirá que no 
precisa de dicho apoyo. Es más: aunque en este estudio no hemos analizado las consecuencias negativas (o daños psicológicos) del teletrabajo, sabemos por la literatura previa que existe una serie de riesgos asociados con un excesivo ajuste al teletrabajo, como es la adicción al trabajo. De este modo, la familia percibiría que el teletrabajador (con alta AET y alto ajuste al teletrabajo), además de no precisar de su apoyo pase demasiadas horas teletrabajando, por lo que su apoyo hacia esta actividad laboral puede disminuir.

\section{Limitaciones del estudio}

Este estudio empírico cuenta con una serie de limitaciones, a continuación planteamos algunas de las principales. En primer lugar, los datos de este estudio se han obtenido de manera voluntaria: se ofreció la posibilidad de participar a un amplio espectro de teletrabajadores. El hecho de que sea una muestra de estas características habría determinado que esté positivamente sesgada, pudiendo darse el caso de que intervinieran los teletrabajadores que poseyeran algunas características (por ejemplo, los teletrabajadores que se sintieran mejor en su trabajo) o motivaciones (curiosidad por los resultados del feedback inmediato), y no otros. Sin embargo, esta forma de recoger datos ha propiciado la hetereogeneidad de estos y, por tanto, amplía su capacidad de generalización. Pero pensamos que sí podría ser interesante comparar los resultados obtenidos con los realizados con una muestra seleccionada al azar (por ejemplo, de una o varias grandes empresas) para homogeneizar este tipo de variables, además de incrementar el número de participantes.

En segundo lugar, existen variables, como la gestión efectiva de la distancia (GED), que necesita mejorar. Así, aunque se decidió mantenerla en el estudio tal y como estaba planteada en su origen para ser fieles a la filosofía de los autores que plantearon su existencia (y debido a que su fiabilidad, aunque no muy elevada, era aceptable) lo cierto es que ha mostrado un bajo poder predictor, y cuando lo ha hecho (con la interferencia de la familia en el teletrabajo), no ha sido en el sentido esperado. Sin embargo, por lo que conocemos, es la primera vez que se desarrolla una medida de este tipo, ajustada a la literatura específica de teletrabajo, que deberá ser validada en estudios posteriores con muestras más amplias.

En tercer lugar, cabe señalar que el modelo planteado no ha explicado de la manera esperada la relación entre el teletrabajo y la vida familiar/personal (ni la interferencia ni el apoyo), aunque sí en el sentido contrario. Esto se debería a la falta de inclusión de variables también negativas (como las demandas) o más relacionadas con la gestión de los recursos humanos a nivel más general (políticas de recursos humanos, estrategias organizacionales).

Por tanto, y a nivel global, podemos 
señalar que el modelo planteado ha sido parcialmente confirmado, comportándose las variables predichas de manera diferente en función a las variables predictoras, sobre todo en relación con el papel principalmente modulador de la AET. De este modo, se ha revelado la importancia de la vía positiva del modelo RED, mostrando la fuerza de los recursos (organizacionales y personales) para explicar las experiencias/ emociones en el teletrabajo, así como el papel fundamental que juega la AET a la hora de explicar la salud psicosocial de los teletrabajadores. Pasemos, pues, a analizar algunas de sus aplicaciones prácticas.

En futuros estudios sería interesante examinar las relaciones causales entre las variables independientes, moduladoras y dependientes analizadas. Para ello, se necesitaría un estudio longitudinal con al menos tres recogidas de datos para poder comprobar si, siguiendo la teoría de la autoeficacia (Bandura, 2002) y los estudios sobre espirales de positividad de la autoeficacia (Salanova, Llorens \& Schaufeli, 2011) encontramos espirales virtuosas en el desarrollo de la salud psicosocial de los trabajadores impulsadas por sus niveles previos de autoeficacia.

Aplicaciones prácticas: Estrategias DE PROTECCIÓN DE LA SALUD PSICOSOCIAL DEL TELETRABAJADOR INTEGRADAS EN LA GESTIÓN Y DESARROLLO DE LOS RECURSOS HUMANOS EN LA EMPRESA
Como ya hemos señalado, el presente trabajo pone de relieve la importancia de fomentar, desde el punto de vista de la psicología ocupacional positiva, estrategias en las organizaciones que potencien la salud psicosocial en el trabajo, y no solo que disminuyan el malestar o el estrés psicosocial. Es decir: desarrollar las ventajas potenciales del teletrabajo a través de la mejora de la salud psicosocial del teletrabajador; función que debería ser fomentada por los responsables de los recursos humanos en las empresas.

Por otro lado, desde la psicología de la salud ocupacional, hemos visto la fuerza que determinados recursos organizacionales e individuales pueden tener sobre la percepción de las experiencias y emociones (salud psicosocial) en el teletrabajo. Y sobre todo, se ha puesto de relieve la importancia de la autoeficacia para el teletrabajo (el creer que tú puedes) a la hora de optimizar la salud psicosocial, así como la importancia de concretar a qué tipo de colectivos se dirigen las actuaciones concretas (por ejemplo, ofrecimiento de mayor cantidad de facilitadores a los teletrabajadores con menores niveles de autoeficacia para el teletrabajo).

De este modo, las acciones dirigidas a la protección de la salud psicosocial del teletrabajador no deberían verse como una acción puntual por parte de la empresa, sino reflejada en todos los procesos clásicos de gestión y desarrollo de recursos humanos. En rela- 
ción con los procesos de gestión, esta integración debería hacerse patente en procesos tales como la planificación de recursos humanos (teniendo en cuenta el número de empleados/as que desearían teletrabajar en el futuro), el análisis y diseño de puestos de trabajo (por ejemplo, dotando los puestos de facilitadores organizacionales o gestionando adecuadamente la distancia), en la selección de potenciales teletrabajadores (considerando su competencia mental, características de personalidad, autoeficacia para el teletrabajo, el apoyo de su familia hacia las prácticas de teletrabajo o su experiencia en la empresa). Por otro lado, las prácticas de desarrollo de recursos humanos han mostrado un papel moderador entre teletrabajo y desempeño (Martínez-Sánchez et al., 2007). En relación con la integración de la salud psicosocial de los teletrabajadores, esta debería hacerse patente en procesos como la formación (incrementando aspectos como el autocontrol, la automotivación, la autoeficacia para el teletrabajo, ser técnicamente autosuficiente o el desarrollo de estrategias de apoyo al teletrabajo que permitan conciliar la vida personal con la laboral) o el desarrollo de la carrera.

Se trata, en definitiva, de que la dirección de la empresa (la dirección de recursos humanos en particular) tenga en cuenta al (tele)trabajador a la hora de configurar la nueva actividad de trabajo, dejándole participar (y negociar) en la definición de las fronteras y el conte- nido del trabajo. Se implantaría el teletrabajo no solo basándose en la tecnología (que permite actualmente realizar cualquier ajuste técnico imaginable con relativa sencillez) sino, y sobre todo, basándose en la persona que va a ocupar ese nuevo puesto. Sería lo que se denomina el lado humano del cambio.

Por todo ello, también se pone en relieve la importancia de la evaluación e intervención en los riesgos psicosociales en esta nueva forma de actividad laboral, pudiéndose encontrar presente de manera integrada en todos los procesos de gestión y desarrollo de los recursos humanos en la empresa. Las diferentes leyes de prevención de riesgos laborales garantizan la salud integral de los trabajadores dondequiera que trabajen o la actividad que desempeñen, incluyendo el teletrabajo. Como señala Jack Nilles (1973): "El teletrabajo no es ni un premio, ni un castigo. Es solo una modalidad diferente de trabajar". Como profesionales de los recursos humanos, el convertirlo en un premio, con sus ventajas para la salud psicosocial, está en nuestras manos.

\section{REFERENCIAS}

Aiken, L. S., \& West, S. G. (1991). Multiple regression: Testing and interpreting interactions. Newbury Park, CA: Sage.

Alonso, M. B., \& Cifre, E. (2002). Teletrabajo y salud: Un nuevo reto para la Psicología. Papeles del Psicólogo, 
83, 55-61.

Bandura, A. (2002). Self-efficacy: The exercise of control. ( $5^{\text {a }}$ edición). Nueva York, NY: Freeman and Company.

Bilbeny, N. (1993). El idiota moral. La banalidad del mal en el siglo XX. Barcelona: Anagrama.

Canadian Telework Asociation (1997). Advanteges and Desvadvanteges of telework for teleworkers. Recuperado el 23 de septiembre del 2001, de http://www.ivc.ca/proteleworkers. html

Cifre, E. (2008). Estrategias de mejora de la salud psicosocial del teletrabajador: El arte de conjugar teoría y práctica. Revista de Trabajo y Seguridad Social Centro Estudios Financieros, 300, 181-200.

Cifre, E., \& Salanova, M. (2004). Estrategias de conciliación familia/trabajo: Buscando la calidad de vida laboral. Estudios Financieros, 259, 111-154.

Cifre, E., Martínez-Pérez, M. D., Salanova, M., Llorens, S., Martínez, I. M., \& Grau, R. M. (2004a). Cuestionario de evaluación de riesgos psicosociales en teletrabajadores " $R E D$ TT”. Registrado el 01/12/2004, con $\mathrm{n}^{\mathrm{o}}$ de asiento registral 09/2004/2416. Conselleria d'Educació, Castellón de la Plana, España.

Cifre, E., Salanova, M., Martínez-Pérez, M. D., Martínez, I. M., Llorens, S., \& Grau, R. M. (2004b). Developing a new tool to assess specific psychosocial risks among teleworkers: The RED-TT questionnaire. 3rd Conference on Occupational Risk Prevention, Santiago de Compostela.

Cifre, E., Salanova, M., \& Rodríguez, A. M. (2006). Tradicional job vs. Teleworking: Psychosocial consequences on mental health. $7^{\text {th }}$ full conference of the European Academy of Occupational Health Psychology, noviembre. Dublín.

Cohen, J., \& Cohen, P. (1983). Applied Multiple Regression/Correlation analisis for the Behavioural Sciences (2 ${ }^{\mathrm{a}}$ ed.). Hillsdale, NJ: Erlbaum.

Conner, M., Fletcher, W., Firth-Cozens, J., \& Collins, S. (1994). Teleworking, stress and health. En H. Schroeder et al. (Eds.), Health psychology: Potential in diversity (pp. 309-313). Regensbrug, Alemania: S. Roeder Verlag.

Demerouti, E., Bakker, A. B., Nachreiner, F., \& Schaufeli, W. B. (2001). The Job Demands-Resources model of burnout. Journal of Applied Psychology, 86, 499-512.

Escobar, M., Jiménez, R., Del Riego, L., \& Rodríguez, J. (1998). Teletrabajo. Incidencia social y económica. Posibilidades en España. Madrid: Escuela de Organización Industrial y Retevisión.

Flanagan, J. C. (1954). The critical incident technique. Psychological Bul- 
letin, 51, 327-355.

Grau, R. M., Salanova, M., \& Peiró, J. M. (2000). Efectos moduladores de la autoeficacia en el estrés laboral. Apuntes de Psicología, 18(1), 57-75.

Hartig, T., Kylin, C., \& Johansson, G. (2007). The telework tradeoff: Stress mitigation vs. Constrained restoration. Applied Psychology: An International Review, 56(2), 231-253.

Hernández-Muñoz, J. M. (1997). Teletrabajo. Nuevos servicios basados en trabajo cooperativo. Recuperado el 19 de abril de 1999, de http://www. selva.dit.upm.es/ proy/doct/Teletrabajo_tt/Teletrabajo_tt/sld023.htm

Ilozor, D. B. e Ilozor, B. D. (2002). Australian telecommuting: Management communication strategies. Logistics Information Management, 15(2), 80-87.

Jaccard, J., Turrisi, R., \& Wan, C. K. (1990). Interaction effects in multiple regression. Newbury Park, CA: Sage.

Karasek, R. A. (1979). Job demands, job decision latitude and mental strain: Implications for job redesign. Administrative Science Quarterly, 24, 285-308.

Karasek, R., \& Theorell, T. (1990). Healthy work: Stress productivity, and the reconstruction of working life. Nueva York, NY: Basic Books.

Kleinbaum, D. G., Kupper, L. L., \&
Muller, K. E. (1988). Applied regression analysis and other multivariate methods ( $2^{\mathrm{a}}$ ed.). Boston: PWS-Kent.

Kowalski, K. B., \& Swanson, J. A. (2005). Critical success factors in developing teleworking programs. Benchmarking: An International Journal, 12(3), 236-249.

Landsbergis, P. A., Schnall, P. L., Warren, K., Pickering, T. G., \& Schwartz, J. E. (1994). Association between ambulatory blood pressure and alternative formulations of job strain. Scandinavian Journal of Work, Environment and Health, 20, 349-363.

Lewis, S. (2003). Flexible working arrangements: Implementation, outcomes, and management. En C. L. Cooper, \& I. T. Robertson (Eds.), International review of industrial and organizacional psychology (pp. 1-28). Chichester, U.K.: John Wiley y Sons, Ltd.

Martínez, I. M., \& Salanova, M. (2005). Obstáculos y facilitadores organizacionalesy su relaciónconel burnoutdocente.prFORM@R. Online. Recuperado el 12 de enero de 2012, de http://www. wont.uji.es/wont/downloads/articulos/ nacionales/2005MARTINEZ05AN. pdf

Martínez, I. M., \& Salanova, M. (2006). Autoeficacia en el trabajo: El poder de creer que tú puedes. Estudios Financieros, 279, 175-202.

Martínez-Sánchez, A., Pérez-Pérez, M., De Luis-Carnicer, P., \& Vela-Jimé- 
nez, M. J. (2007). Telework, human resource flexibility and firm performance. New Technology, Work and Employment, 22(3), 208-223.

Nilles, J. (1973). The telecomunicationstransportation tradeoff. Options for tomorrow and today. California: Jala Internacional.

Nunnally, J. C., \& Bernstein, I. H. (1994). Psychometric theory, $3^{\text {a }}$ ed. Nueva York: McGraw-Hill.

Padilla, A. (1997). Teletrabajo, dirección y organización. Madrid: Editorial Rama.

Peeters, M. C. W., Buunk, B. P., \& Schaufeli, W. B. (1995). Social interactions and feelings of inferiority among correctional officers: A daily event-recording approach. Journal of Applied Social Psychology, 25, 1073-1089.

Peters, P., Tijdens, K. G., \& Wetzels, C. (2003). Employees' opportunities, preferences, and practices in telecommuting adoption. Information \& Management, 41, 469-482.

Pérez, M., Martínez, A., \& De Luis, P. (2003). The organizational implications of human resources managers' perception of teleworking. Personnel Review, 32(6), 733-755.

Pérez, M. P., Sánchez, A. M., \& De Luis, M. P. (2002). Benefits and barriers of telework: Perception differences of human resources managers according to company's operations strate- gy. Technovation, 22, 775-783.

Pérez, J., Sancho, T., \& Nogareda, C. (1996). NTP 412: Teletrabajo: Criterios para su implantación. Nota Técnica de Prevención. INSHT. Recuperado el 18 de diciembre del 2006, de http://www.mtas.es/insht/ ntp/ntp_412.htm

Preacher, K. J. (2007). A primer on interaction effects in multiple linear regression. Recuperado el 15 de enero del 2007, de http://www. psych.ku.edu/preacher/interact/interactions.htm

Raghuram, S., Garud, R., Wiesenfeld, B. M., \& Gupta, V. (2001). Factors contributing to virtual work adjustment. Journal of Management, 27(3), 383-405.

Salanova, M., Bresó, E., \& Schaufeli, W. B. (2005). Hacia un modelo espiral de las creencias de eficacia en el estudio del burnout y del engagement. Ansiedad y Estrés, 11(2-3), 215-231.

Salanova, M., Llorens, S., \& Schaufeli, W. B. (2011). Yes, i can, i feel good $\&$ i just do it! On gain cycles and spirals of efficacy beliefs, affect, and engagement. Applied Psychology: An International Review, 60(2), 255-285.

Salanova, M., Cifre, E., Martínez, I. M., \& Llorens, S. (2007). Caso a caso en la prevención de los riesgos psicosociales. Metodología WoNT para 
una organización saludable. Bilbao: Lettera publicaciones.

Schaufeli, W. B., \& Bakker, A. B. (2004). Job demands, job resources and their relationship with burnout and engagement: A multi-sample study. Journal of Organizational Behavior, 25, 293-315.

Schneider, B., \& Bowen, D. (1993). The service organization: Human resources management is crucial. Organizational Dynamics, 21, 39-52.

Schneider, B., White, S. S., \& Paul, M. C. (1998). Linking service climate and customer perceptions of service quality: Test of a causal model. Journal of Applied Psychology, 83, 150-163.

Schwarzer, R. (1999). General perceived self-efficacy in 14 cultures. Self-Efficacy assessment. Recuperado el 3 de julio del 2003, de http:// www.yorku.ca/faculty/academic/ schwarze/world14.htm

Speier, Ch., \& Frese, M. (1997). Generalized self-efficacy as a mediator and moderator between control and complexity at work and personal initiative: A longitudinal field study in East Germany. Human Performance, 10, 171-192.

Tausig, M., \& Fenwick, R. (2001). Unbinding time: Alternate work schedules and work-life balance. Journal of Family and Economic Issues, 22(2), 101-119.
Tesluk, P. E., \& Mathieu, J. E. (1999). Overcoming roablocks to effectiveness: Incorporating management of performance barriers into models of work group effectiveness. Journal of Applied Psychology, 84(2), 200-202. 